\title{
Left ventricular restoration for ischemic cardiomyopathy and simultaneous implantation of left ventricular assist system actively aiming at bridge to recovery
}

Goro Matsumiya, MD, Yuji Miyamoto, MD, Osamu Monta, MD,

Hiroshi Takano, MD, Yoshiki Sawa, MD, and Hikaru Matsuda, MD, Osaka, Japan

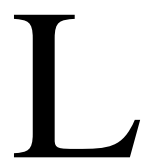

eft ventricular restoration (LVR) is a useful treatment option for ischemic cardiomyopathy (ICM). However, its current indication is limited for those with relatively stable congestive heart failure symptoms and preserved end-organ function. ${ }^{1,2}$ We report the case of a patient with ICM with cardiogenic shock and end-organ dysfunction who underwent LVR and implantation of a left ventricular assist system (LVAS) as a scheduled operation actively aiming at bridge to recovery.

\section{Clinical Summary}

A 31-year-old man with Marfan syndrome had Stanford type A aortic dissection, which involved the left main trunk. Although he successfully underwent valve-sparing aortic root replacement, a large myocardial infarction developed. In the next 3 months his condition progressed to New York Heart Association class IV congestive heart failure. Despite multiple inotropic supports, hemodynamic measurements showed a systolic pulmonary artery pressure of $55 \mathrm{~mm} \mathrm{Hg}$ and cardiac index of $1.6 \mathrm{~L} \cdot \mathrm{min} \cdot \mathrm{m}^{2}$. Echocardiogram (Figure 1) showed a left ventricular (LV) diastolic/systolic dimension of $71 / 64 \mathrm{~mm}$, a left ventricular ejection fraction of $19 \%$, and moderate mitral regurgitation. Cardiogenic shock, oliguria, and hepatic dysfunction (total bilirubin $5.6 \mathrm{mg} / \mathrm{dL}$ ) eventually developed in the patient, and he was brought to an operating room.

With the use of cardiopulmonary bypass (CPB) and cardioplegic cardiac arrest, mitral annuloplasty was performed using a 26-mm Physio ring (Edwards Lifesciences, Irvine, Calif). Through the longitudinal left ventriculotomy, a Dacron patch $(2.5 \mathrm{~cm}$ wide $)$ was sutured to the margin of the infarcted area at the septum and lateral wall. The ventriculotomy line was closed over the patch leaving the apical area open for the LVAS cannula. An outflow graft was sutured to the side of the graft at the ascending aorta. With

\footnotetext{
From the Division of Cardiovascular Surgery, Department of Surgery, Osaka University Graduate School of Medicine, Osaka, Japan.

Received for publication Sept 7, 2004; revisions received Nov 1, 2004; accepted for publication Nov 3, 2004.

Address for reprints: Goro Matsumiya, MD, Department of Surgery, Osaka University Graduate School of Medicine (E1), 2-2 Yamadaoka, Suita, Osaka, 565-0871, Japan (E-mail: matsumg@surg1.med.osaka-u.ac.jp).

J Thorac Cardiovasc Surg 2005;130:219-20

$0022-5223 / \$ 30.00$

Copyright (C) 2005 by The American Association for Thoracic Surgery

doi:10.1016/j.jtcvs.2004.11.003
}

an extracorporeal-type LVAS (Toyobo-NCVC, Nipro, Japan) support, the patient was successfully weaned from CPB.

The patient's end-

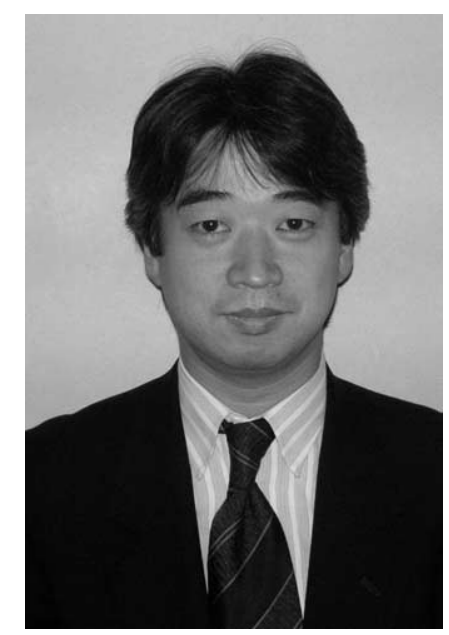

Dr Matsumiya organ function recovered gradually; echocardiographic parameters (Figure 1) and brain natriuretic peptide level improved with time (Figure 2). Cardiac function was assessed 70 days after the operation. During the temporal LVAS halt, hemodynamic parameters showed no deterioration, and left ventricular ejection fraction and cardiac index improved with the increasing dose of dobutamine. After 78 days of support, the LVAS was explanted successfully. Although there was a transient increase of brain natriuretic peptide, the echocardiogram showed consistent improvement of LV function after the LVAS explantation (Figure 2). He is doing well with New York Heart Association class II symptoms at 1 year since the LVAS removal.

\section{Discussion}

Early experiences of LVR for ICM demonstrated poor outcomes in emergency cases complicated with cardiogenic shock and/or end-organ dysfunction. ${ }^{1}$ In such cases, the implantation of an LVAS and subsequent heart transplantation are generally indicated. The bridge use of an LVAS for myocardial recovery is reported in patients with idiopathic dilated cardiomyopathy, but patients with ICM have little chance of recovery with only an LVAS.

There would be 2 scenarios in which patients with ICM experience cardiogenic shock. ${ }^{2}$ One is acute myocardial infarction in the remaining viable areas. The other is progressive remodeling and resultant failure of remaining myocardium. In the latter scenario, LVR has a potential power to recover LV function. However, end-organ dysfunction after cardiogenic shock is often hard to overcome by the restored LV that has only marginal function. An LVAS can afford potent circulatory support for a prolonged period and provide a good chance of end-organ recovery.

There may be several other treatment options. First, intra-aortic balloon pump and/or extracorporeal life support as a short-term assist after the LVR may have the limited power to reverse the end-organ dysfunction. The other is the implantation of an LVAS followed by the LVAS removal together with the LVR. With this strategy, it may be difficult to assess the reversibility of the LV 


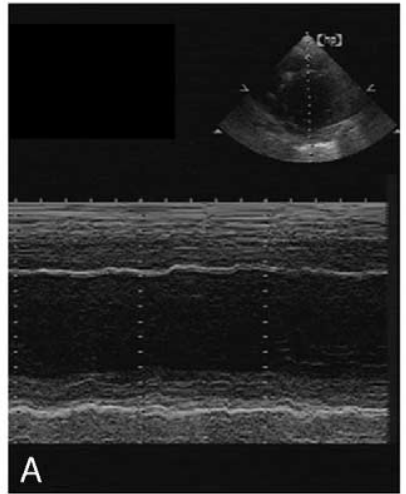

LVDd $71 \mathrm{~mm}$

FS $\quad 10 \%$

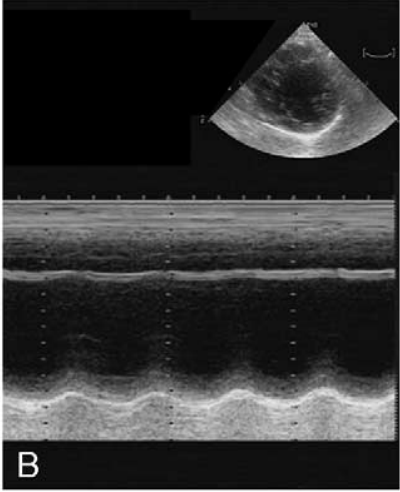

LVDd 62mm

FS $14 \%$

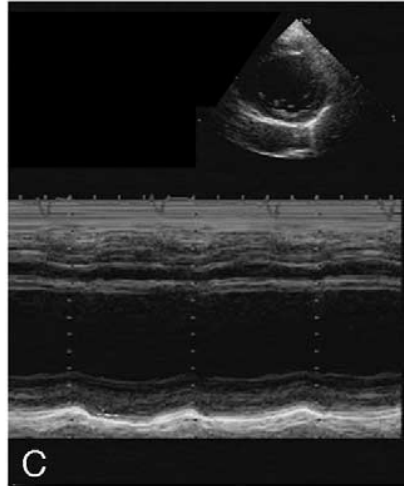

LVDd $65 \mathrm{~mm}$

FS $13 \%$

Figure 1. Short-access and M-mode view of the left ventricle (LV) by echocardiogram (A) before the left ventricular restoration (LVR) and left ventricular assist system (LVAS) implantation, (B) just before the successful LVAS removal, and (C) at the sixth month after the LVAS removal. The reduction of diastolic dimension and improvement of contraction at the posterior wall were noted after the operation and persisted after the LVAS explantation. LVDd, Left ventricular end-diastolic dimension; FS, fractional shortening.

Changes of BNP and Echocardiographic parameters

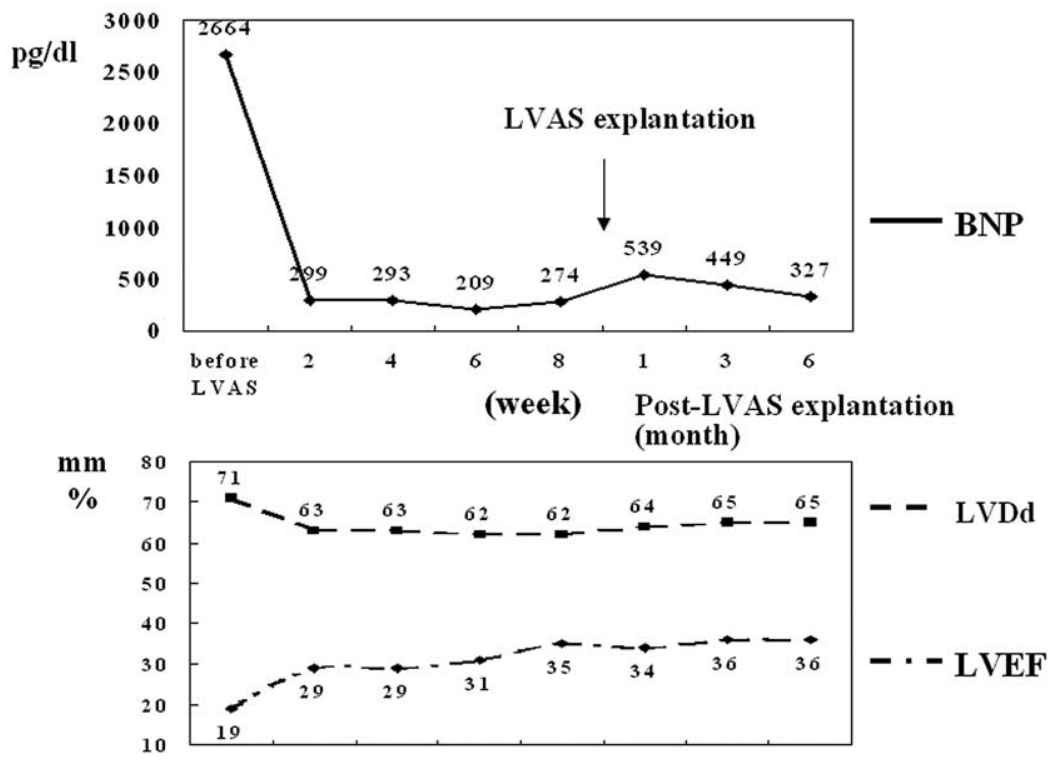

Figure 2. Change of serum brain natriuretic peptide level and echocardiographic parameters before and after the LVR and LVAS implantation and after the LVAS explantation. LVDd, Left ventricular end-diastolic dimension; $L V E F$, left ventricular ejection fraction.

during the LVAS support. Furthermore, the use of cardiac arrest and $\mathrm{CPB}$ at the LVAS removal may cause significant damage to the impaired myocardium.

In summary, simultaneous LVR and LVAS implantation aiming at myocardial recovery is one of the treatment options for ICM complicated with cardiogenic shock and end-organ dysfunction.

\section{References}

1. Bolooki H, DeMarchena E, Mallon SM, et al. Factors affecting late survival after surgical remodeling of left ventricular aneurysms. J Thorac Cardiovasc Surg. 2003;126:374-85.

2. McCarthy PM. Ventricular aneurysms, shock, and late follow-up in patients with heart failure. J Thorac Cardiovasc Surg. 2003;126:323-5. 